\title{
Extracting Dominant Turbulent Structures in Supersonic Flow Using Two-Dimensional Fourier Transform
}

Toshinori Kouchi (Corresponding author)

Dept. Mechanical and System Engr., Okayama University

Tsushimanaka 3-1-1, Kita, Okyama, Okayama 700-8530, Japan

Tell: +81-86-251-8045 / Fax: +81-86-251-8045

E-mail: kouchi@okayama-u.ac.jp

Goro Masuya

Dept. Mechanical and Aerospace Engr., Tohoku University

Aoba 6-6-01, Aramaki, Aoba, Sendai, Miyagi 980-8579, Japan

Shinichiro Yanase

Dept. Mechanical and System Engr., Okayama University

Tsushimanaka 3-1-1, Kita, Okyama, Okayama 700-8530, Japan

\begin{abstract}
A new image process for quantifying both convection velocities $(U \mathrm{c})$ and scales $\left(\lambda_{d}\right)$ of turbulent structures captured in a fast-framing schlieren movie is presented. We obtained 90 time-series schlieren images of a transverse jet into a Mach 2 supersonic flow with 1-MHz sampling. The schlieren images captured not only the shock and expansion waves but also the turbulent structures within the jet and the boundary layer. The image intensities were extracted along the outer edges of the jet and the boundary layer and were remapped as a time-space intensity map. The time-space map exhibited swept stripe patterns, indicating that stable turbulent structures were periodically generated and convected downstream. The angle and interval of the stripe patterns were efficiently extracted using the two-dimensional Fourier transform, which corresponded to the $U \mathrm{c}$ and $\lambda_{\mathrm{d}}$ of the dominant structures. The zero-padding fast Fourier transform and the sub-pixel estimation of the spectral peak positions in the Fourier domain improved the accuracy for evaluating the angle and interval of the stripes, which resulted in the accurate evaluation of $U \mathrm{c}$ and $\lambda_{\mathrm{d}}$. The proposed method was validated by comparing the $U \mathrm{c}$ obtained using the proposed method to those obtained via schlieren image velocimetry for both the transverse jet and the supersonic boundary layer.
\end{abstract}




\section{List of Symbols}

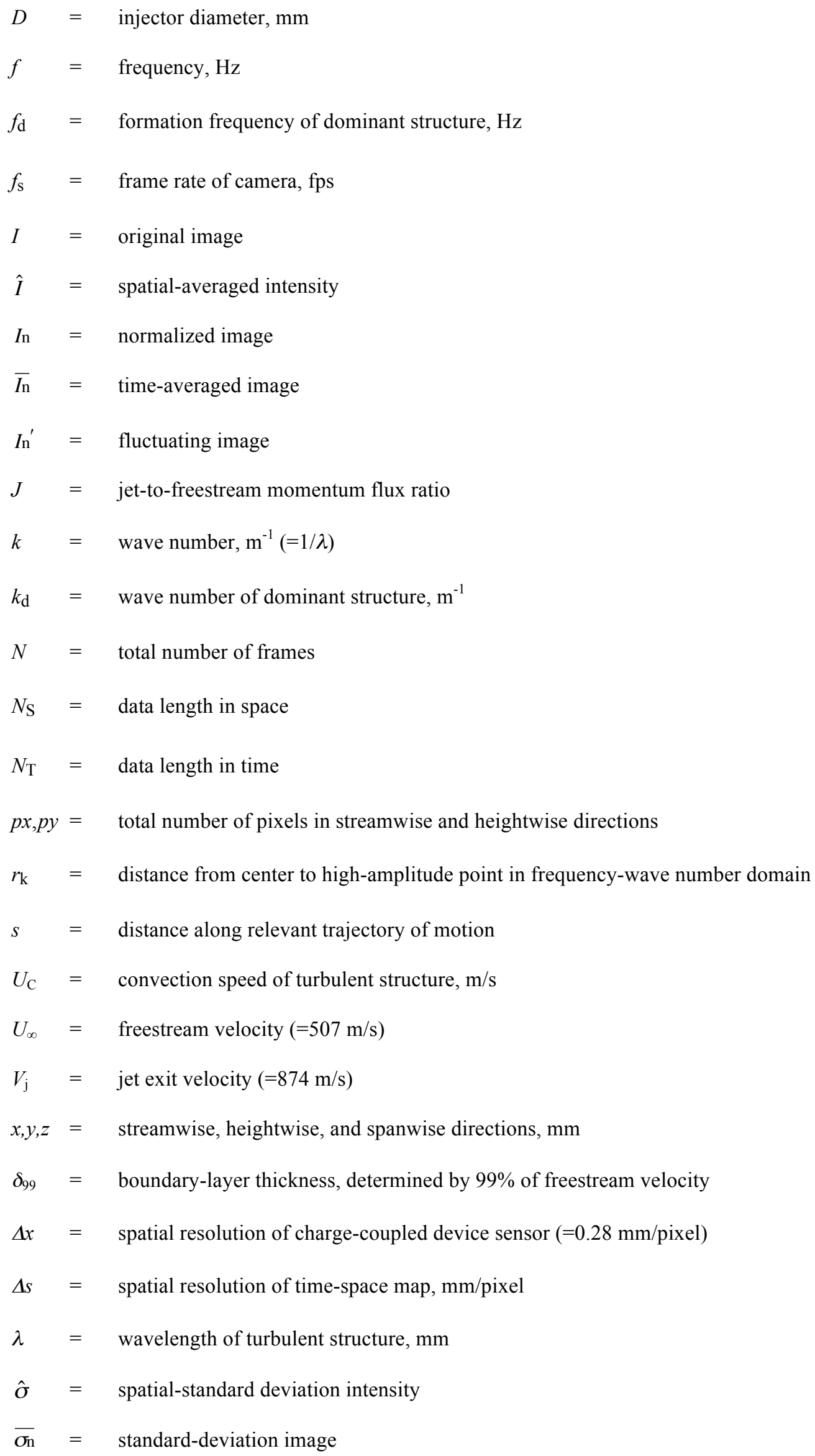




$$
\begin{aligned}
& \theta=\text { angle of stripe pattern on time-space map, }{ }^{\circ} \\
& \theta_{\mathrm{k}}=\text { angle to high-amplitude point in two-dimensional power spectrum, }{ }^{\circ}
\end{aligned}
$$

\title{
Subscripts and Superscripts
}

\author{
$n \quad=\quad$ frame number \\ $i, j \quad=\quad$ streamwise and heightwise pixel numbers of schlieren image
}

\section{Introduction}

Unsteady measurements in supersonic and hypersonic flows require measuring instruments having a very high-frequency response, typically in the rage of several hundred kilohertz to several megahertz. Because such high-frequency response instruments are difficult to obtain, unsteady measurement is very challenging for supersonic and hypersonic flows. In the low-speed flow regime, constant temperature-type hot-wire anemometers (CTAs) are often used to measure the fluctuating velocity. The frequency response of a CTA is usually in the rage of several tens of kilohertz to $100 \mathrm{kHz}$, which is not high enough for supersonic and hypersonic flows. Comte-Bellot and Sarma (2001) developed a constant voltage-type hot-wire anemometer (CVA) having a response of several hundreds of kilohertz for a supersonic flow. Recently, Kondo et al. (2009 \& 2010) measured the fluctuations of the mass flux and concentration of a fuel jet in a supersonic flow using a parallel double-hot-wire probe and a CVA circuit. The hot-wire method provides only point-wise information. Planar and volumetric measurements, which are usually based on flowvisualization techniques, are preferable for investigating the flow structures in detail.

Fast-framing schlieren imaging is one of the powerful visualization techniques for unsteady high-speed flows. It is straightforward compared with other methods, such as particle image velocimetry (PIV), planar Doppler velocimetry (PDV), planar laser Rayleigh/Mie scattering (PLS), planar laser-induced fluorescence (PLIF), and pressure-sensitive paint (PSP). Fast-framing visualization requires a high-intensity light source for capturing instantaneous flow features and a high repetition rate for capturing a time-series image. However, the scattering light from particles and fluorescence and phosphorescence from molecules, which are used for PIV, PDV, PLS, PLIF and PSP, are weak. 
Bueno et al. (2005) and Ganapathisubramani et al. (2006 \& 2007) captured the motion of a turbulent structure within the supersonic boundary layer via PIV and PLS, with a $10-\mathrm{kHz}$ repetition Nd:YLF laser. The repetition rates of their measurements were low because they focused on the low-frequency motion of the flow separation due to the shock wave-oundary layer interaction. Their measurements indicated that very large-scale structure motion (VLSM) occurred in the supersonic boundary layer, similar to the structures in the subsonic boundary layer (Kim \& Adrian 1999), and that the VLSM significantly affected the size of the shock-induced separation and its low-frequency motion. The repetition rate of available commercial lasers is on the order of $1-10 \mathrm{kHz}$, which imposes a limitation on unsteady imaging.

The development of a pulse-burst laser system (Thurow et al. 2013) allowed megahertz-rate PIV, PDV, PLS, and PLIF. The remarkable resulting unsteady-imaging data revealed the turbulent structure motions in supersonic flows and provided insight into not only mechanism of supersonic turbulent mixing but also the shock wave-boundary layer interaction. Unfortunately, the pulseburst laser system is still difficult to create by only researchers in fluid dynamics.

On the other hand, schlieren imaging requires only a simple, inexpensive, and weak illumination system compared with the previous ones. In schlieren imaging, light from the source is directly focused on an imaging sensor, whereas in many other techniques, weak illumination from particles or molecules is measured. Therefore, the intensity of schlieren images is far higher than that of images obtained using other techniques, and schlieren imaging is easier than the other techniques for fast-framing visualization. Although schlieren imaging has great advantages for the illumination system, the technique typically employs qualitative measurement. Quantitative information must be extracted from schlieren images.

Some researchers focused on the turbulent structures captured in schlieren or shadowgraph images and evaluated their convection velocity. The convection velocity is one of the important indices for the effect of the compressibility on the growth rate of a jet. Therefore, these data are useful for developing scramjet combustors, which require rapid mixing between fuel and air because of the limited time and combustor length.

For subsonic flows, Brown and Roshko (1974) investigated the convection velocity in subsonic mixing layers. They captured a shadowgraph movie using a high-frame rate camera to examine the unsteady motions of eddies in the subsonic mixing layer. They remapped the eddy 
locations as time-space map to investigate the eddy formation, amalgamation events, and convection velocity.

For a supersonic flow, Smith and Smits (1995) observed the large-scale coherent structures in a supersonic turbulent boundary layer via cinematographic schlieren imaging and other visualization techniques. They graphically evaluated the convection velocity by using the $x-t$ diagram of the sequential binary schlieren images. They concluded that the convection velocity of the boundary-layer thickness-size structures in the boundary layer was $0.9 \pm 0.1$ of the freestream flow speed. Jonassen et al. (2006) and Hargather et al. (2011) evaluated the convection velocity in a supersonic boundary-layer and jet from two schlieren images using the same manner of PIV. Their system combined commercially available PIV equipment capable of $15-\mathrm{Hz}$ acquisitions with a standard schlieren optical instrument. This imaging system captured structures far smaller than the boundary-layer thickness-size structures in the boundary layer. The convection velocity of the small structures followed the local flow speed in the boundary layer.

Ben-Yakar et al. (2002 \& 2006) captured the time evolution of a jet into a supersonic flow by using fast-framing schlieren images. They measured the convective velocity of turbulent eddies in a jet into a supersonic crossflow from eight time-series schlieren images with a typical sampling rate of $1 \mathrm{MHz}$. They tracked the displacement of the individual eddies using two-frame imagebased fast Fourier transform (FFT) cross-correlation, which is similar to PIV. In their method, the interrogation area was selected manually to include the eddy of interest. They also investigated the formation cycle of the eddy manually. The manual selection was difficult when the numbers of eddies and frames increased. Manual detection may introduce uncertainty because of the arbitrary choice.

To investigate the relationship between the convection velocity and the growth rate of eddies, information regarding the eddy size and the convection velocity should be extracted from the image data. The size of the eddy depends on the eddy amalgamation. Therefore, we developed a new method for non-manually extracting information regarding both the size and convection velocity of eddies from the fast-framing images. We focused on the image gradations due to the eddies in the image and remapped the image intensities of the eddies as a time-space map in the similar manner to Brown and Roshko (1974), Smith and Smits (1995), and Ben-Yakar et al. (2002 $\&$ 2006). The method uses a two-dimensional (2D) Fourier transform to efficiently extract the dominant structures. 
In the present study, we applied the newly proposed technique to a Mach 2 supersonic boundary layer and a sonic transverse injection into a Mach 2 supersonic flow. This paper begins with a description of the wind-tunnel facility, including the flow conditions. A detailed description of the proposed method is then presented, followed by the results. The combination of the zeropadding fast Fourier transform (FFT) for a 2D Fourier transform and the sub-pixel estimation of the peak findings in the Fourier domain remarkably improved the spatial resolution of the analysis. For the case of the supersonic boundary layer, the convection velocities obtained using the proposed method were compared with previously published experimental data (Spina et al. 1991; Poggie et al. 2004; Hargather et al. 2011). They were also compared with data obtained using image-based cross-correlation (Gruber et al. 1997; Ben-Yakar et al. 2002) for both the supersonic boundary layer and the transverse jet.

\section{Experimental Apparatus}

The experiments were conducted in a suction-type supersonic wind-tunnel facility, which is schematically depicted in Fig. 1. A 2D contoured nozzle inhaled unheated atmospheric air into the test section. The nominal facility Mach number was 2.0 . The test section had a $30 \times 30-\mathrm{mm}^{2}$ cross section, and its length was $274 \mathrm{~mm}$. Fused silica glass windows were set on the sidewalls. An injector module was mounted flush on the bottom wall of the test section, $184 \mathrm{~mm}$ downstream from the nozzle exit. The incoming boundary layer reached a thickness of $\delta_{99}=3.6 \mathrm{~mm}$ at the injector position. Its momentum thickness was $0.3 \mathrm{~mm}$ (Uramoto et al. 2016). The Reynolds number based on the momentum thickness and the freestream conditions was approximately $4.0 \times$ $10^{3}$

The injector module consisted of a sonic injector block and a manifold. The orifice diameter (D) was $1.0 \mathrm{~mm}$, and its depth was $12.5 \mathrm{~mm}$. A miniature piezoelectric pressure transducer was installed on the manifold to monitor the stagnation pressure of the jet. The stagnation pressure was used to evaluate the jet-to-crossflow momentum flux ratio $(J)$.

We conducted two series of experiments: transverse-jet experiments and supersonic boundarylayer experiments. For the transverse-jet experiments, room-temperature helium-simulating fuel hydrogen — was injected perpendicularly through a sonic orifice into the freestream. In this study, $J$ was 6.0 , and the Reynolds number based on $D$ and the jet conditions was $3.0 \times 10^{4}$. For the 
supersonic boundary-layer experiments, we acquired schlieren movies without the injection. A Cartesian coordinate system was used to represent the results, with the origin at the center of the injector, the streamwise direction along the $x$-axis, the height from the injector wall along the $y$ axis, and the spanwise direction along the $z$-axis.

A mirror-based, $z$-arrangement schlieren system captured the turbulent structures and the shock waves in the flow field. A Shimadzu HPV-1 fast-framing camera $(312 \times 260$ pixel array $)$ was used to capture fast-framing schlieren movies. The camera recorded 90 time-series images at 1,000,000 frames per second (fps) in a single experiment. The exposure time of each frame was 500 ns. A strobe flash for a camera (Panasonic PE-60SG) with a pulse duration of approximately $1 \mathrm{~ms}$ was used as the light source. Two $f / 10,200-\mathrm{cm}$ focal length concave mirrors collimated the light from the source and refocused it onto a knife edge. The knife edge was oriented along the heightwise direction of the test section to emphasize the density gradients in the streamwise direction.

The field of view for the imaging was approximately $90 \mathrm{~mm} \times 30 \mathrm{~mm}$, as indicated by the shaded area in Fig. 1. A calibration target image ( $5 \mathrm{~mm} \times 5 \mathrm{~mm}$ grid board) was captured before each test run, compensating for the run-to-run discrepancy of the imaging. The size of each pixel in the images corresponded to $0.28 \mathrm{~mm}$. The fluid elements shifted approximately $0.25 \mathrm{~mm}$ downstream with 500-ns exposure when the flow speed was $500 \mathrm{~m} / \mathrm{s}$, which corresponds to a Mach 2 flow. The shift value was smaller than a pixel. Thus, the present imaging system captured instantaneous flow structures.

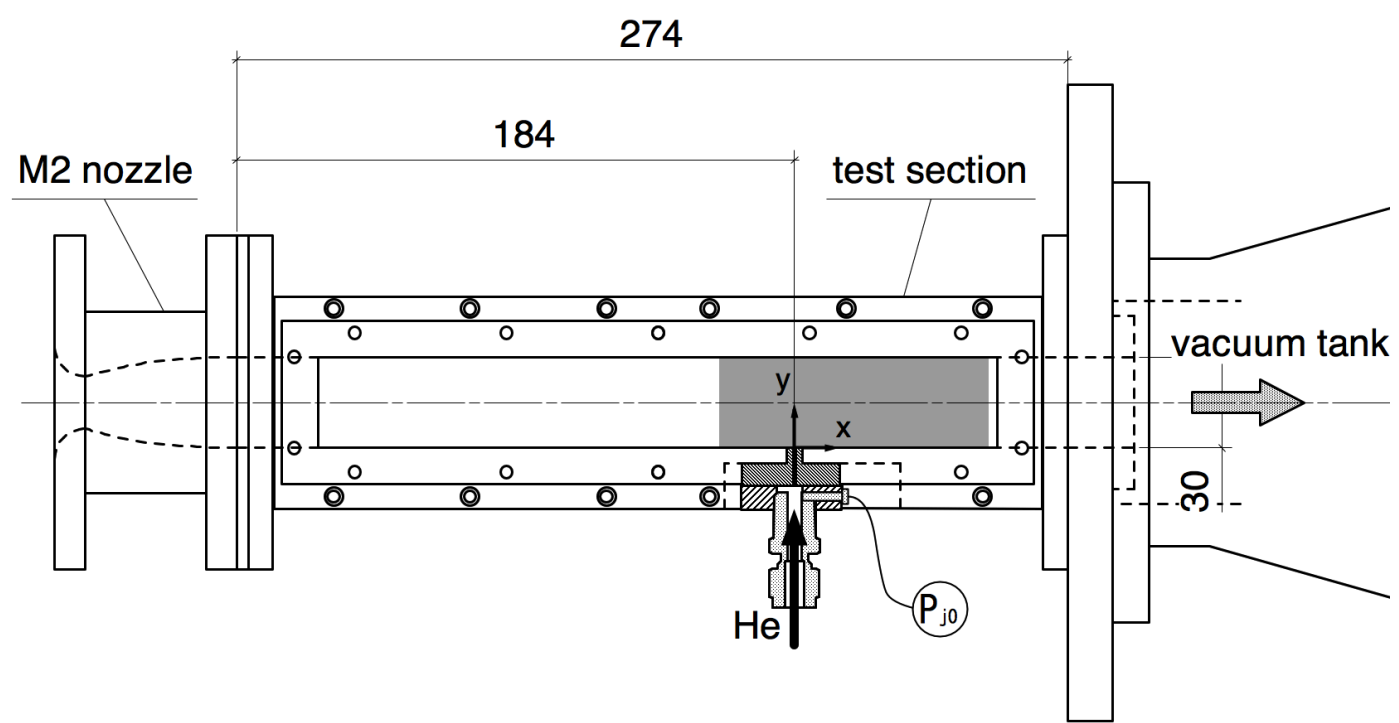

Fig. 1 Schematic of the suction-type supersonic wind tunnel with a test section. 


\section{Image Analysis}

Figure 2 summarizes the image processing for extracting the convective velocity and scale of the dominant turbulent structures from a time-series of schlieren images. The image processing mainly comprised three parts: the enhancement of the turbulent structures, the conversion of the time-series images into a time-space map, and 2D Fourier analysis. The details of each process are discussed below.

\subsection{Enhancement of Turbulent Structures}

First, we normalized the original image $\left(I_{i j}^{n}\right)$ using the spatial-averaged intensity $\left(\hat{I}^{n}\right)$ and the spatial-standard deviation of the image intensities $\left(\hat{\sigma}^{n}\right)$ for the region of interest, where the subscripts $i$ and $j$ represent the streamwise and heightwise pixel numbers of the image, respectively, and the superscript of $n$ represents the number of frames. The normalized image $\left(\operatorname{In}_{i j}^{n}\right)$ is given by

$$
\operatorname{In}_{i j}^{n}=\frac{1}{\hat{\sigma}^{n}}\left[I_{i j}^{n}-\hat{I}^{n}\right]
$$

where $\hat{I}^{n}$ and $\hat{\sigma}^{n}$ are computed as

$$
\begin{gathered}
\hat{I}^{n}=\frac{1}{p x \cdot p y} \sum_{i=1}^{p x} \sum_{j=1}^{p y} I_{i j}^{n} \\
\hat{\sigma}^{n}=\sqrt{\frac{1}{p x \cdot p y} \sum_{i=1}^{p x} \sum_{j=1}^{p y}\left[I_{i j}^{n}-\hat{I}^{n}\right]^{2}}
\end{gathered}
$$

This normalization was performed under the assumption that all the images had similar structures throughout the test. This normalization reduced the error due to light-intensity fluctuations. The series of $\operatorname{In}_{i j}^{n}$ yields the following series of fluctuating images $\left(\operatorname{In}_{i j}^{n^{\prime}}\right)$ :

$$
\operatorname{In}_{i j}^{n^{\prime}}=\operatorname{In}_{i j}^{n}-\overline{\operatorname{In}_{i j}}
$$

where $\overline{\operatorname{In}_{i j}}$ is the time-averaged image computed as

$$
\overline{\mathrm{In}_{i j}}=\frac{1}{N} \sum_{n=1}^{N} \operatorname{In}_{i j}^{n}
$$

Here, $N$ is the total number of frames. 


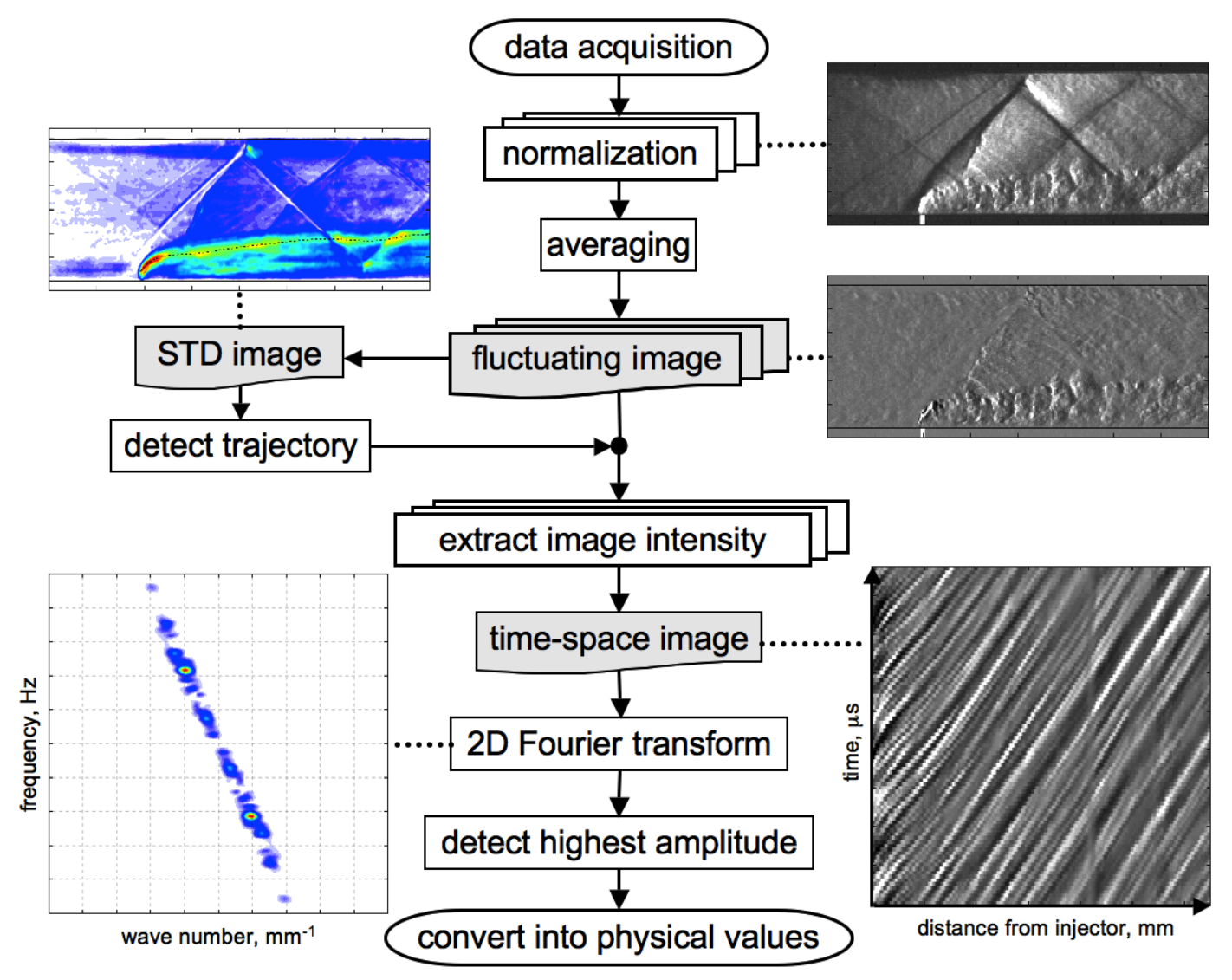

Fig. 2 Image-analysis process.

Figure 3 shows one of the typical schlieren images for the transverse-injection experiments, which was normalized with respect to $\hat{I}^{n}$ and $\hat{\sigma}^{n}$. The main stream traveled from left to right, while the injectant entered perpendicularly from the bottom wall. This picture well captured the typical flow features of the transverse-injection experiments: turbulent structures in the jet, bow shock wave due to the injection, a recompression shock wave, and pressure waves emanating from the jet boundary.

Figure 4 shows the fluctuating image corresponding to Fig. 3. The subtraction of $\overline{\mathrm{In}_{i j}}$ from $\mathrm{In}_{i j}^{n}$ emphasized the moving structures, such as the turbulent eddies in the jet and pressure waves from the jet boundary. On the other hand, it eliminated the steady structures, such as the weak shock waves from the facility nozzle and the bow shock wave due to the injection. The turbulent structures in the jet were clearly represented by the periodical change in the image intensity. Figure 5 shows the intensity along the turbulent structures framed by the dashed lines in Fig. 4. The intensity along the turbulent structures was nearly sinusoidal. A positive intensity was observed in the left portion of a structure, and a negative intensity was observed in the right 
portion of the structure. The scales of the turbulent structures were estimated by counting the cycles of dark and bright bands in the image.

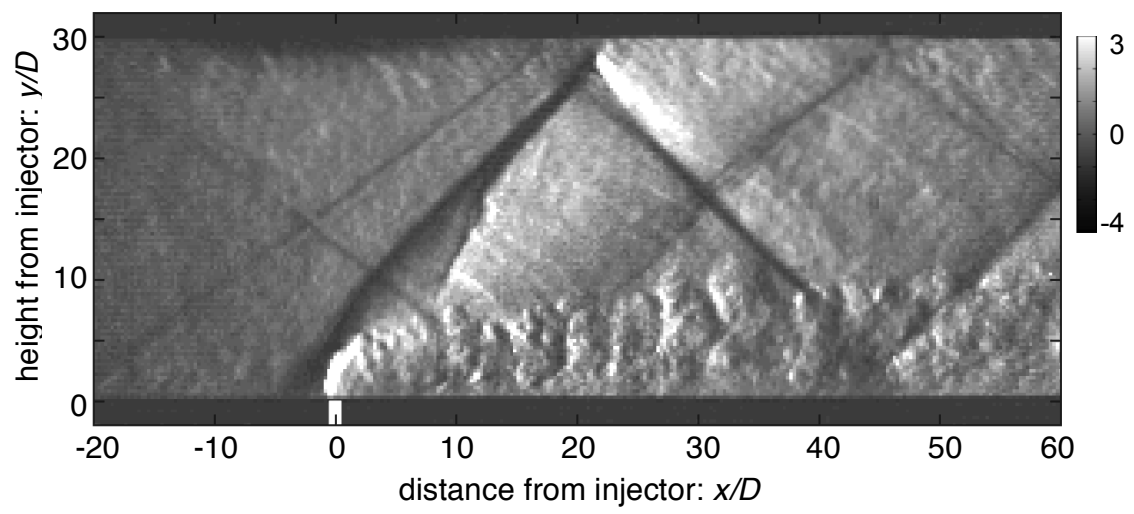

Fig. 3 Typical normalized schlieren image of a transverse jet in a

Mach 2 supersonic flow.

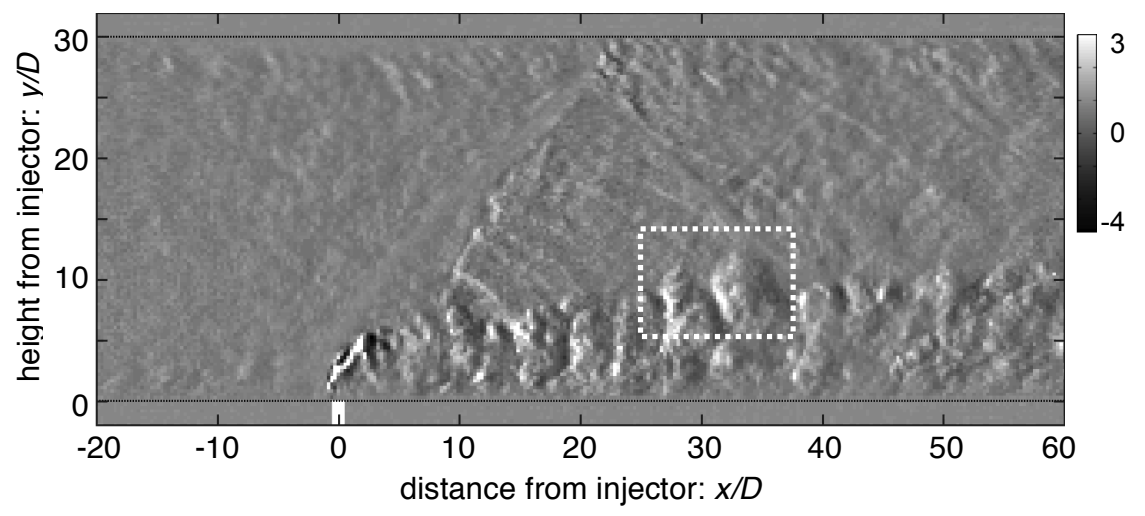

Fig. 4 Typical fluctuating intensity image corresponding to Fig. 3.

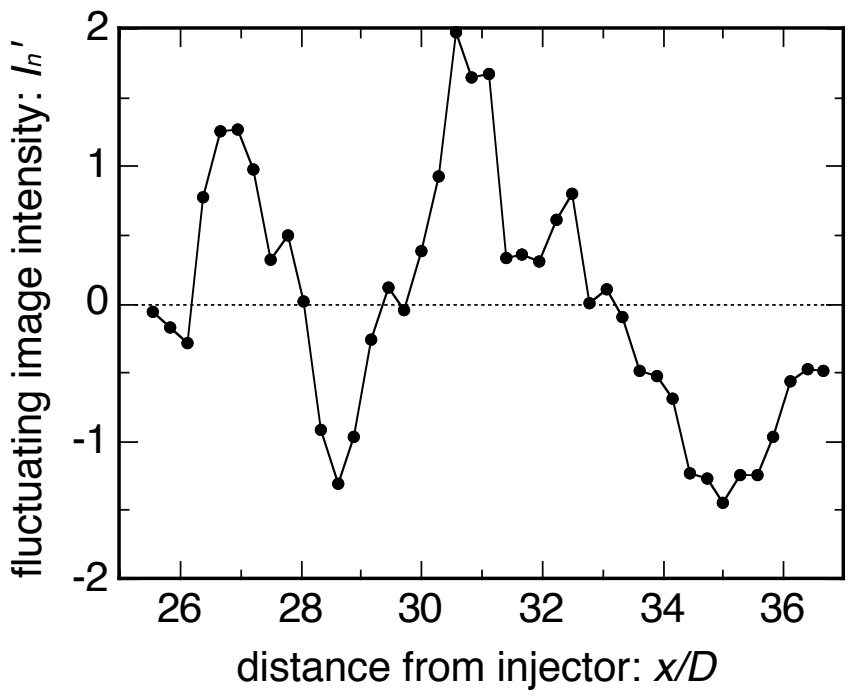

Fig. 5 Fluctuating image intensities on turbulent structures in the transverse jet enclosed by the dashed lines in Fig. 4. 


\subsection{Conversion of Time-Series Images into Time-Space Map}

The trajectory of motion is one of the important factors for analyzing the motion of objects. Usually, the trajectory of motion is automatically determined when the convection velocity of the object is evaluated, because the trajectory is tangential to the direction of the convection velocity. The image-based spatial cross-correlation method (Gruber et al. 1997; Ben-Yakar \& Hanson 2002) for evaluating the convection velocity of turbulent structures automatically determines the trajectory of motion of a turbulent structure. However, the present method using 2D Fourier analysis requires the predetermined trajectory of motion for generating a time-space trajectory map of the turbulent-structure convection. In the present study, we employed a standard-deviation image calculated using a series of the fluctuating images to determine the relevant trajectory of the motion. The standard-deviation image was computed as follows:

$$
\overline{\sigma_{i j}}=\sqrt{\frac{1}{N} \sum_{n=1}^{N} I_{i j} n^{\prime 2}}
$$

Figure 6 shows the standard-deviation image. The standard deviation along the outer edge of the jet was higher than that along the inner layer. The bow shock wave impinged at $x / D \approx 22$ on the top wall. The reflected shock wave impinged into the jet at $x / D \approx 40$ and passed through the jet at $x / D \approx 52$. Downstream from the shock waves, $\overline{\sigma_{n}}$ was increased. The shock impingement increased the fluctuation. In the present image analysis, we tracked the maximum standard deviation along the outer edges of the jet as a representative trajectory where the turbulent structures were convected, as indicated by the dashed lines in Fig. 6. The trajectory of interest was chosen arbitrarily for the present analysis. When interested in the inner region of the jet or the boundary layer, one can choose other trajectories related to the inner motion. In the present study, we mainly focused on the structure along the outer edge of the jet. The validity of this trajectory for the jet motion is discussed in detail in Sec. 4.2.

The image intensities were sampled along the jet trajectory and reconstructed in a time-space intensity map. Figure 7 shows the time-space intensity map reconstructed from a time-series of the fluctuating images. The horizontal axis indicates the distance along the jet trajectory from the injector hole $(s)$ normalized with respect to $D$, and the vertical axis indicates the image-acquisition time. The map clearly shows right-running stripe patterns. The stripe patterns indicate that stable turbulent structures were periodically generated and convected downstream. The convection 
velocity of the structure is indicated by the angle of the stripes, and the size of the structure was calculated using the horizontal interval of the stripes.

Figure 7 indicates that the angle and interval of the stripes differed with respect to $x / D$. For $x / D$ $=5-10$, the angle was smaller and the interval was narrower than those downstream. Both the angle and interval increased as the structures travelled downstream to $x / D \approx 20$. For $x / D>20$, there was no remarkable change in the angle or interval. These trends of the angle and interval imply that small structures were generated near the fuel injector and convected downstream at a high speed. The small structures traveled downstream, merging to form larger structures. The convection speed of the larger structures decreased and approached a specific value.

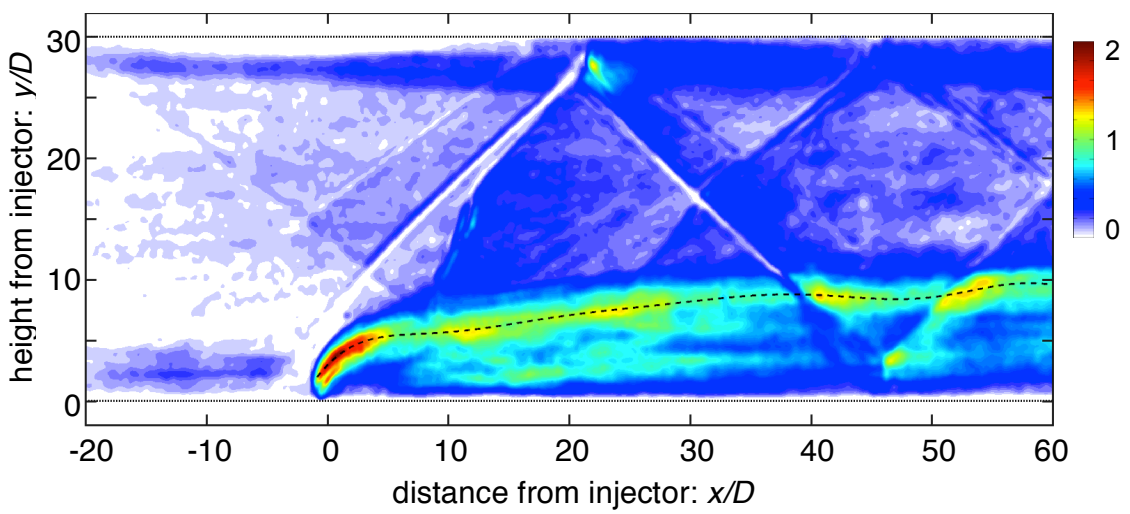

Fig. 6 Standard-deviation image.

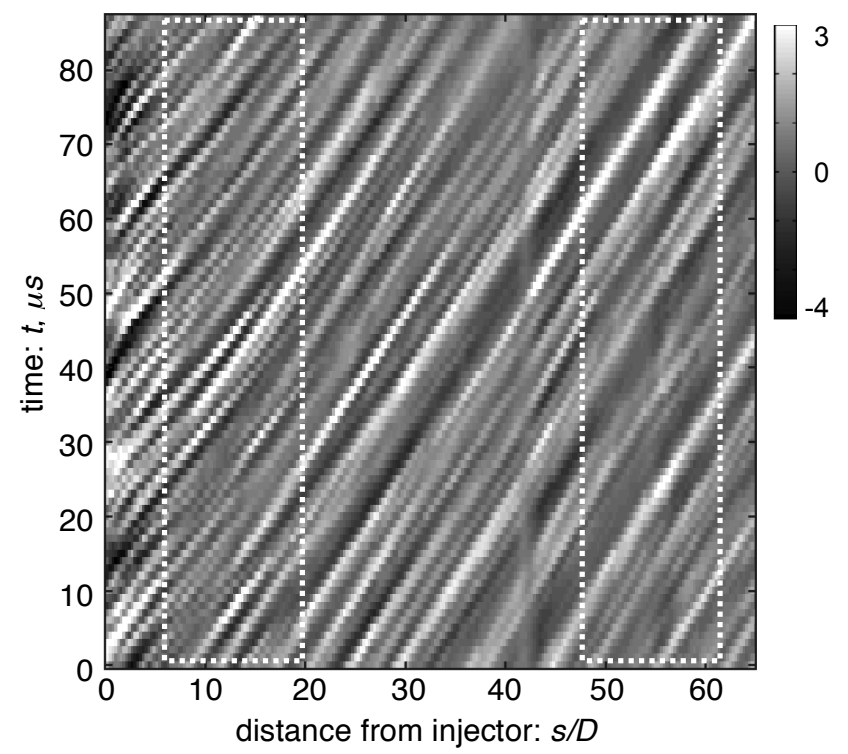

Fig. 7 Time-space map of the fluctuating intensity on the outer edge of the jet. 


\subsection{D Fourier Analysis}

A 2D Fourier transform efficiently extracts the features on a time-space map. When the 2D Fourier transform is applied to diagonal stripes, the $2 \mathrm{D}$ power spectrum shows two high-amplitude points corresponding to the angle and interval of the diagonal stripes. These two points have point symmetrical with respect to the image center (DC component). To clarify the nature of the 2D Fourier transform, the power spectrum for some time-space diagonal stripe patterns is shown in Fig. 8. The horizontal axis indicates the wave number $(k)$ normalized with respect to the inverse of the spatial resolution on the time-space map $(1 / \Delta s)$, and the vertical axis indicates the frequency $(f)$ normalized with respect to the frame rate $\left(f_{\mathrm{s}}\right)$. These values of $1 / \Delta s$ and $f_{s}$ for the normalization are the inverse values of the scaling factors in the time-space domain. The ranges for each axis are the non-dimensional Nyquist frequency and a wave number of $\pm 1 / 2$.

A high-amplitude point is generated in a direction perpendicular to the stripe pattern. The distance from the image center to the high-amplitude point in the normalized frequency space $\left(r_{\mathrm{k}}\right)$ is inversely proportional to the interval of the stripes in the physical space. The angle to the highamplitude point in the wave-number domain $\left(\theta_{\mathrm{k}}\right)$ corresponds to the stripe angle in the spatial domain: $\theta=\pi / 2-\theta_{\mathrm{k}}$.

The convection velocity of the dominant structures $\left(U_{\mathrm{c}}\right)$ is evaluated according to $\theta_{\mathrm{k}}($ or $\theta)$, as follows:

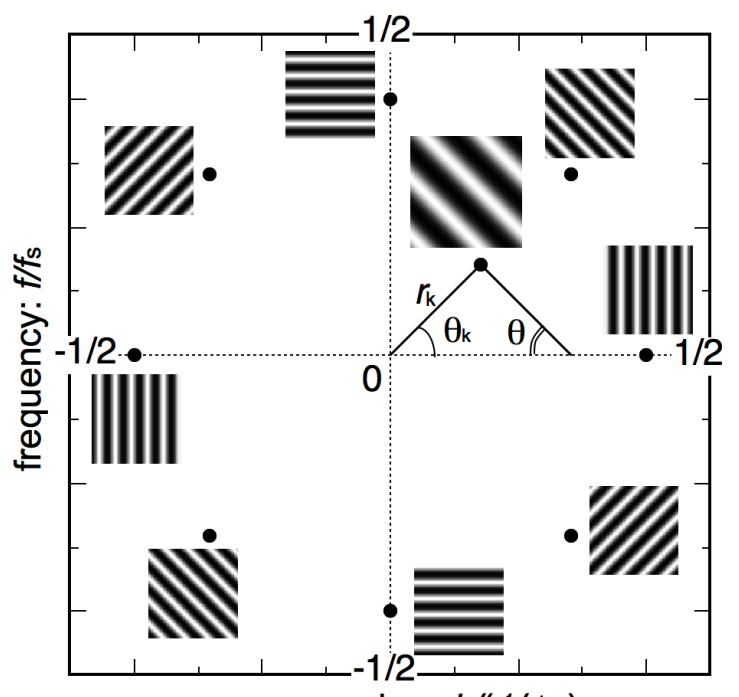

wave number: $k /(1 / \Delta s)$

Fig. 8 Typical response of the 2D Fourier transform for diagonal stripe patterns. 

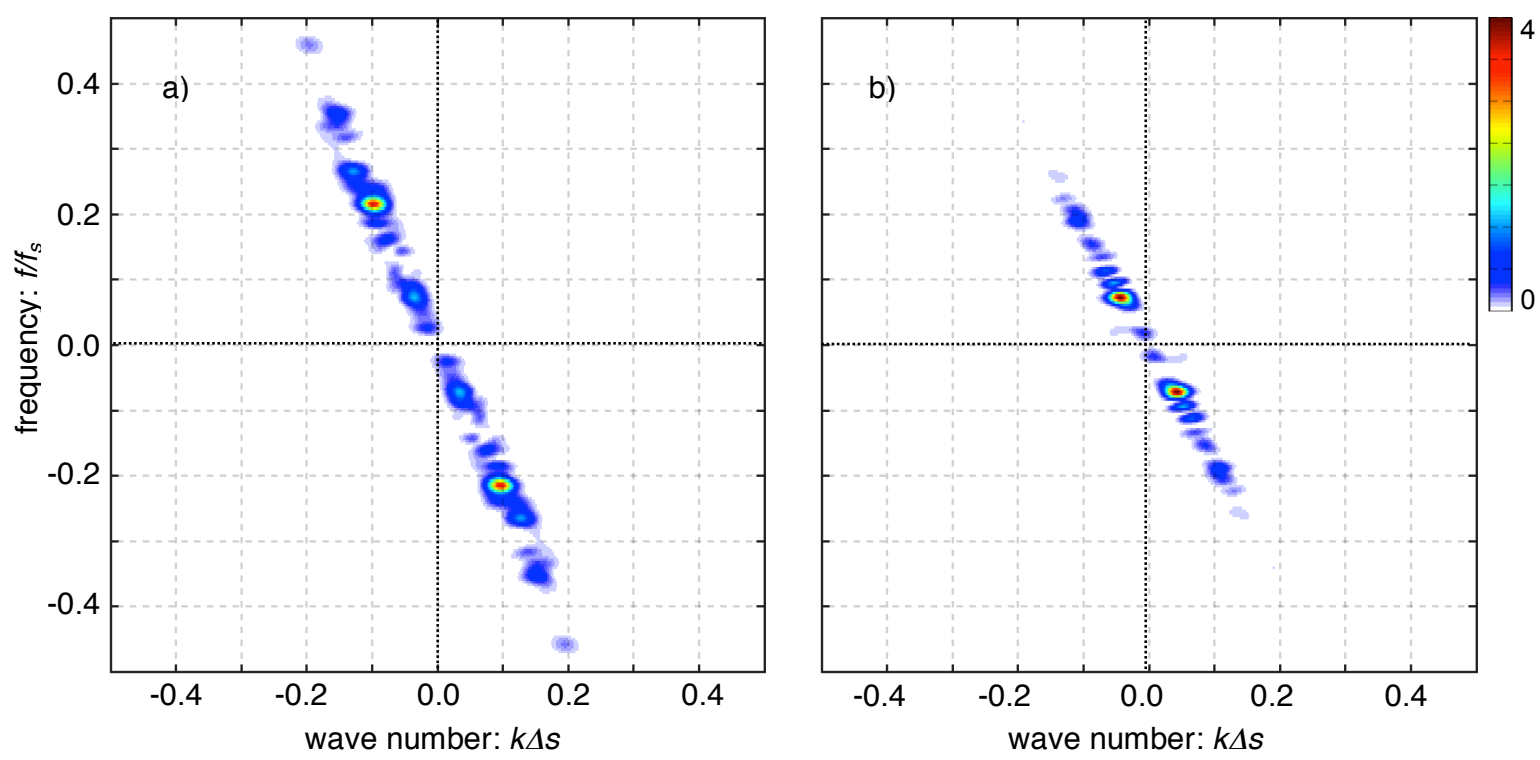

Fig. 9 Frequency-wave number spectra of the image intensity in the jet trajectory: a) near field and b) far field of the injector.

$$
U_{\mathrm{C}}=\frac{\Delta s}{1 / f_{\mathrm{s}}} \frac{1}{\tan \theta}=\Delta s f_{\mathrm{s}} \tan \theta_{\mathrm{k}}
$$

The wave number and formation frequency of the dominant structures $\left(k_{\mathrm{d}}\right.$ and $\left.f_{\mathrm{d}}\right)$ are evaluated according to $\theta_{\mathrm{k}}$ and $r_{\mathrm{k}}$, as follows:

$$
\begin{aligned}
& k_{\mathrm{d}}=\frac{1}{\Delta s} r_{\mathrm{k}} \cos \theta_{\mathrm{k}} \\
& f_{\mathrm{d}}=f_{\mathrm{s}} r_{\mathrm{k}} \sin \theta_{\mathrm{k}}
\end{aligned}
$$

Figure 9 shows the 2D power spectra evaluated from the time-space map shown in Fig. 7. Figures $9 \mathrm{a}$ and $9 \mathrm{~b}$ show the spectra for the regions of $x / D=6-20$ and $x / D=48-62$, as framed by the dashed lines in Fig. 7. These power spectra were obtained with the zero-padding FFT, which is described in detail later. A comparison between Figs $9 \mathrm{a}$ and $9 \mathrm{~b}$ clearly reveals that $\theta_{\mathrm{k}}$ was larger and $r_{\mathrm{k}}$ was longer for the near field of the injector. In the near field, $\theta_{\mathrm{k}}$ was $63.5^{\circ}$, and $r_{\mathrm{k}}$ was 0.24 . In the far field, $\theta_{\mathrm{k}}$ decreased to $59.0^{\circ}$, and $r_{\mathrm{k}}$ was approximately half of that in the near field. According to these $\theta_{\mathrm{k}}$ and $r_{\mathrm{k}}$ values, we evaluated the convection velocity, wave number, and formation frequency of the dominant structures using Eqs. 7, 8, and 9, respectively. 


\subsection{Improvement of Peak Detection}

The accuracy of the estimates for $U_{\mathrm{c}}, k_{\mathrm{d}}$, and $f_{\mathrm{d}}$ depends on the accuracy of the detection of the peak power position in the frequency-wave number domain. The resolution in a frequency space depends on the data length in the real space. To increase the accuracy of the peak detection, a large data length is preferable. However, this reduces the spatial resolution of the analysis. We must divide the full-size time-space image into smaller parts having a short interval of $x / D$ in order to increase the spatial resolution of the analysis. In this subsection, we investigate the relationship between the data length in the spatial domain $\left(N_{\mathrm{S}}\right)$ and the accuracy of estimation for $\theta_{\mathrm{k}}$ and $r_{\mathrm{k}}$ in the Fourier domain.

We used an artificial stripe pattern with an angle of $60^{\circ}$ and a wavelength of 20 pixels to investigate the relationship between $N_{\mathrm{S}}$ and the accuracy of the estimation. The data length in the time direction $\left(N_{\mathrm{T}}\right)$ was the same as $N$, and $N_{\mathrm{S}}$ was changed from 3 to 300 . The artificial image had the similar stripe pattern for the transverse-jet experiments. The theoretical $U_{\mathrm{C}}$ and wavelength $\lambda$ were $477 \mathrm{~m} / \mathrm{s}$ and $5.5 \mathrm{~mm}$ for the experimental conditions of $\Delta s=0.28 \mathrm{~mm} /$ pixel and $f_{\mathrm{s}}=1 \mathrm{Mfps}$.

Figure 10 shows the relationship between $N_{\mathrm{S}}$ and the accuracy of the $U_{\mathrm{C}}$ estimation. The horizontal axis indicates $N_{\mathrm{S}}$ obtained using the Fourier transform, and the vertical axis indicates the relative error of the estimated $U_{\mathrm{C}}$. The dotted lines represent the case without interpolations, and the broken and solid lines represent the cases with interpolations, which are mentioned later.

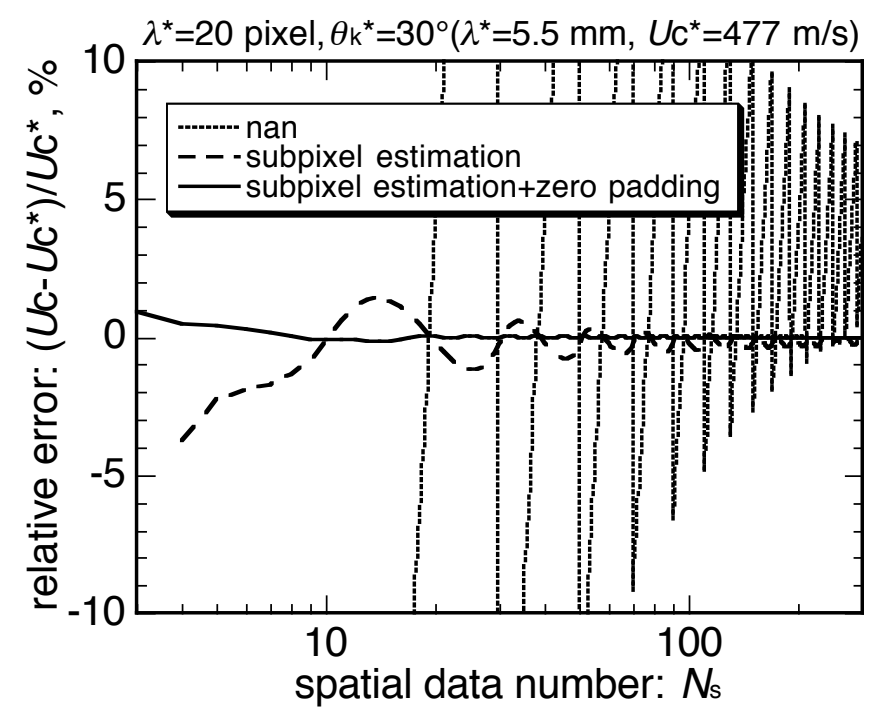

Fig. 10 Accuracy of estimation of the peak position in the Fourier domain using sub-pixel estimation and zero padding. 

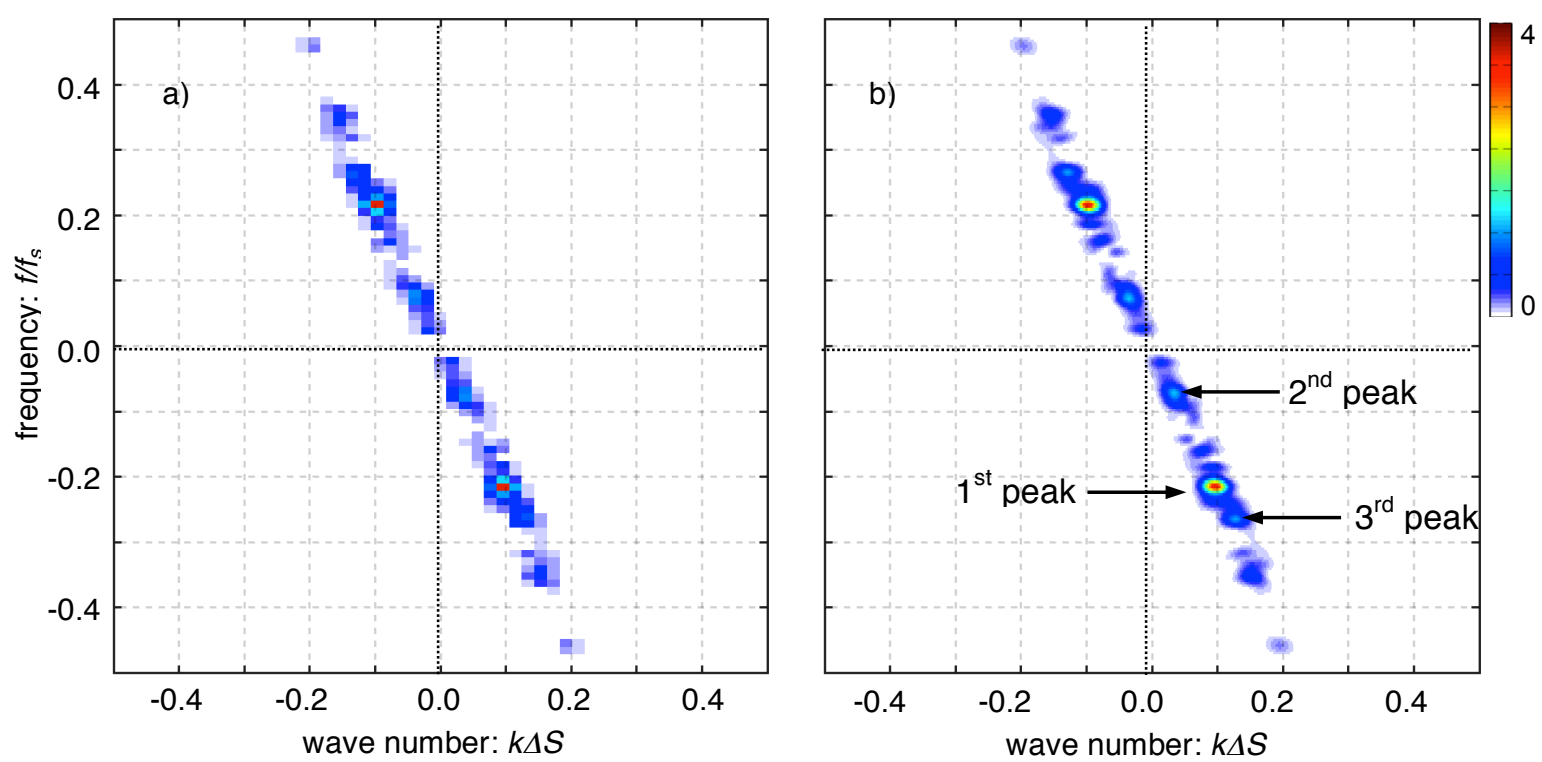

Fig. 11 Effect of zero padding on the frequency-wave number power spectrum: a) without zero padding and $b$ ) with zero padding.

For the case without interpolations, the relative error fluctuated and decreased with increasing $N_{\mathrm{S}}$. However, the relative error was still approximately $+4 \%$ at $N_{\mathrm{S}}=300$. This means that the maximum $N_{\mathrm{S}}$ given by the imaging sensor element yielded insufficient estimation accuracy with no interpolation. To increase the accuracy of the peak detection beyond the sensor size, we applied sub-pixel estimation, which is usually used in PIV (Willert \& Gharib 1991), for detecting the peak power position in the Fourier domain.

The sub-pixel position was estimated using the common method of fitting a Gaussian function to the samples. The dashed lines in Fig. 10 indicate the relative error obtained using the sub-pixel estimation. The relative error in this case was smaller than that in the case without interpolations. The error rapidly approached zero, resulting in $\pm 1 \%$ for $N_{\mathrm{S}}>30$. The data length $N_{\mathrm{S}}$ of 30 corresponds to a spatial resolution of approximately $9 \mathrm{~mm}$ for the present imaging.

To significantly improve the spatial resolution, we appended zeroes to the stripe pattern before taking the Fourier transform (zero-padding FFT) (Lourenco \& Krothapalli 1995). When a signal is padded with zeroes, the data length for the Fourier transform increases with the number of zeroes. Thus, a power spectrum with a substantially higher-frequency resolution is obtained. Figure 11 shows the effects of the zero padding on the 2D power spectrum. Figures 11a and $11 \mathrm{~b}$ show the cases without and with padding, respectively. The data length in the $s$ direction was increased threefold by the zero padding. These power spectra were obtained from the time-space map shown in Fig. 7. The zero-padding yielded improvements in the detection of the spectral peaks-not only 
the highest peak but also the second- and third-highest peaks. The solid line in Fig. 10 indicates the relative error obtained using the zero-padding FFT combined with the sub-pixel estimation for the peak findings. The error in this case was extremely small: $\pm 1 \%$ for $N_{\mathrm{S}}<10$. The trends of the relative errors for $k_{\mathrm{d}}$ and $f_{\mathrm{d}}$ were similar to that for $U_{\mathrm{C}}$.

The padding with the Gaussian sub-pixel interpolation yielded remarkable accuracy improvements, even for $N_{\mathrm{S}}<\lambda$. The periodical data in the time direction compensated for the lack of data in the $s$ direction for $N_{\mathrm{S}}<\lambda$. The small $N_{\mathrm{S}}$ increased the locality and spatial resolution in the analysis. This is one of the significant advantages of the image analysis. For the present study, $N_{\mathrm{S}}$ was set as 15 , and the total data length for the Fourier transform was increased threefold by the zero padding compared with $N_{\mathrm{S}}$.

\section{$4 \quad$ Validations and Discussions}

\subsection{Supersonic Boundary Layer}

Figure 12 shows a typical normalized schlieren image for the supersonic boundary-layer experiment. The horizontal axis indicates the distance from the nozzle exit, and the vertical axis indicates the height from the lower wall. The image shows that the typical scale of the structures in the heightwise direction was approximately $3 \mathrm{~mm}$ at the entrance of the test section and increased to approximately $5 \mathrm{~mm}$ at the exit of the test section. The image also shows that the interfacial wavelength between the boundary layer and the freestream in a certain area appeared very long; for example, $\lambda$ was approximately $10 \mathrm{~mm}$ for $x_{\text {nozzle }}=170-200 \mathrm{~mm}$ and approximately $2 \mathrm{~mm}$ for $x_{\text {nozzle }}>200 \mathrm{~mm}$ for the lower wall. This is because of the VLSM in the boundary layer (Kim \& Adrian 1999; Ganapathisubramani et al. 2006). Uramoto et al. (2016) measured the velocity in the boundary layer in our test section by using PIV and revealed that VLSM occurred on the walls.

The convection velocities for each height were evaluated using the proposed method with the zero-padding 2D FFT, as shown in Fig. 13. The horizontal axis indicates the convection velocity normalized with respect to the freestream velocity of $507 \mathrm{~m} / \mathrm{s}$, and the vertical axis indicates the height from the wall normalized with respect to $\delta_{99}=3.6 \mathrm{~mm}$. The convection velocities were evaluated for the highest, second-highest, and third-highest peaks on the upper and lower walls at

$x_{\text {nozzle }}=184 \mathrm{~mm}$ and were averaged. The error bar indicates the standard deviation of data at the 
same position. We adopted only the second and third peaks that were 0.8 times as large as the highest peak for the averaging. The data were compared with the flow velocity measured using PIV at the same $x_{\text {nozzle }}=184 \mathrm{~mm}$ (Uramoto et al. 2016).

The mean value of the convection velocity at $y / \delta_{99}>0.5$ was $U_{\mathrm{C}} / U_{\infty} \approx 0.85$. The error bar rapidly increased with $y / \delta_{99}$. This was caused by the intermittency in the turbulent boundary layer. Hargather et al. (2011) reported a similar phenomenon in measurements based on image crosscorrelation-like PIV. The method is sometimes called schlieren image velocimetry (SIV). In our experiment, the maximum image fluctuation $\overline{\sigma_{n}}$ was observed at $y / \delta_{99}=0.67$. For a far higher $y / \delta_{99}(>0.67)$, the turbulent structures were sparse, and the dispersion of $U_{\mathrm{C}}$ increased. For $0.2 \leq$ $y / \delta_{99} \leq 0.5$, the measured data were close to the flow velocity. For $y / \delta_{99}<0.2$, the $U_{\mathrm{C}}$ was slightly higher than the flow velocity.

Hargather et al. (2011) reported that their SIV measurements in a supersonic boundary layer agreed with the flow velocity measured in the Pitot survey, except in the region where the intermittency was remarkable. However, our measurements do not reproduce the flow velocity for $y / \delta_{99}<0.2$. It is well-known that the convection velocity obtained via global flow visualization has a nearly constant transverse profile of $U_{\mathrm{C}} / U_{\infty} \sim 0.9 \pm 0.1$ (Spina et al. 1991; Poggie et al. 2004). Poggie and Leger (2015) suspect that the large-scale structures move at a speed of $U_{\mathrm{C}} / U_{\infty} \approx 0.9$; on the other hand, the small-scale structures move at the local mean velocity. The spatial resolution of our schlieren image was coarse for capturing the entire region of the flow field. Therefore, only large-scale structures were captured, resulting in the reproduction of the nearly constant transverse profile of $U_{\mathrm{C}}$ in the boundary layer.

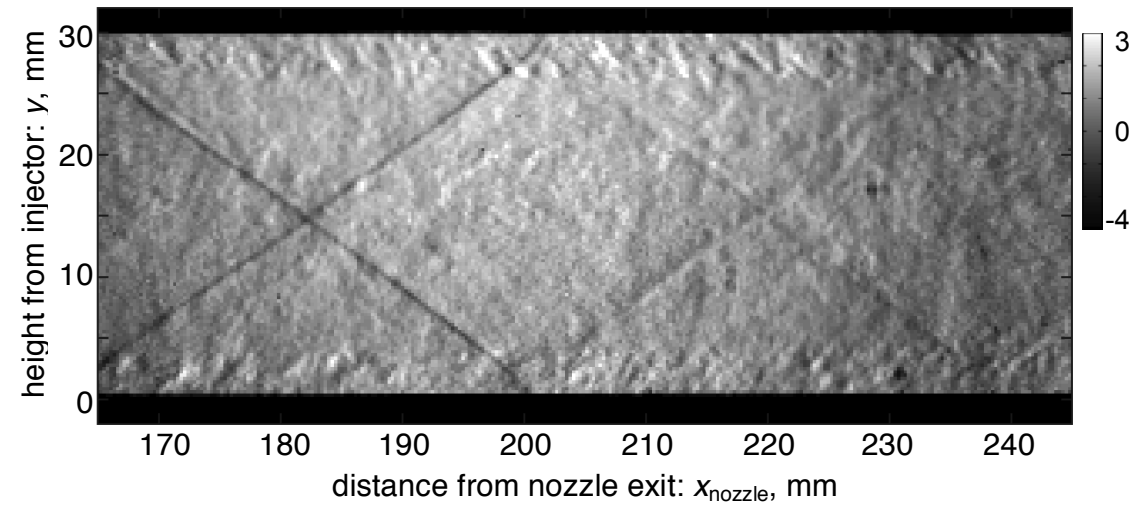

Fig. 12 Typical normalized schlieren image of a supersonic boundary layer. 


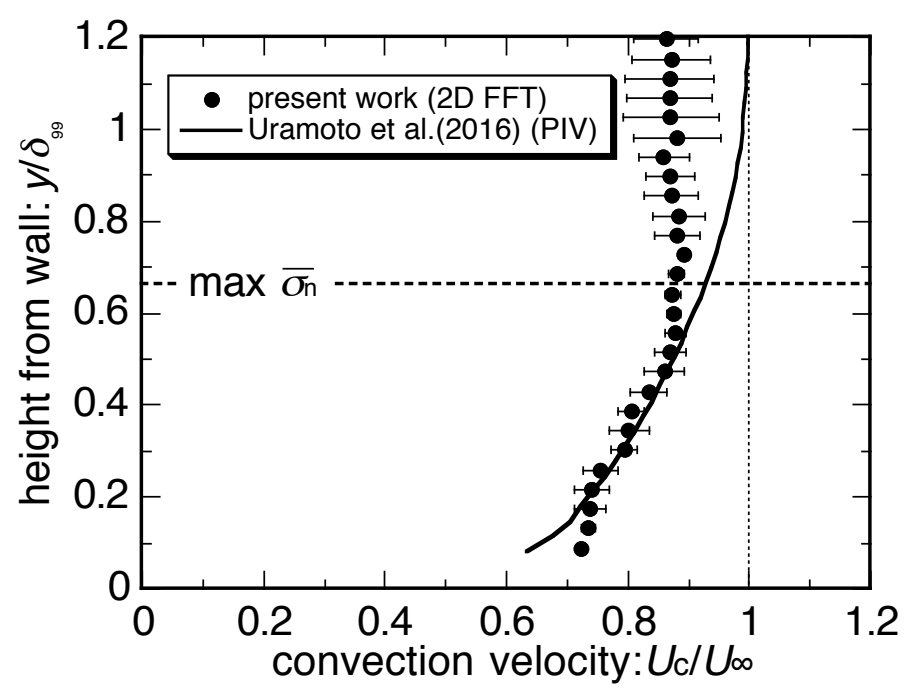

Fig. 13 Comparison of convection velocity evaluated via the zeropadding FFT to the flow velocity measured via PIV in a Mach 2 supersonic boundary layer.

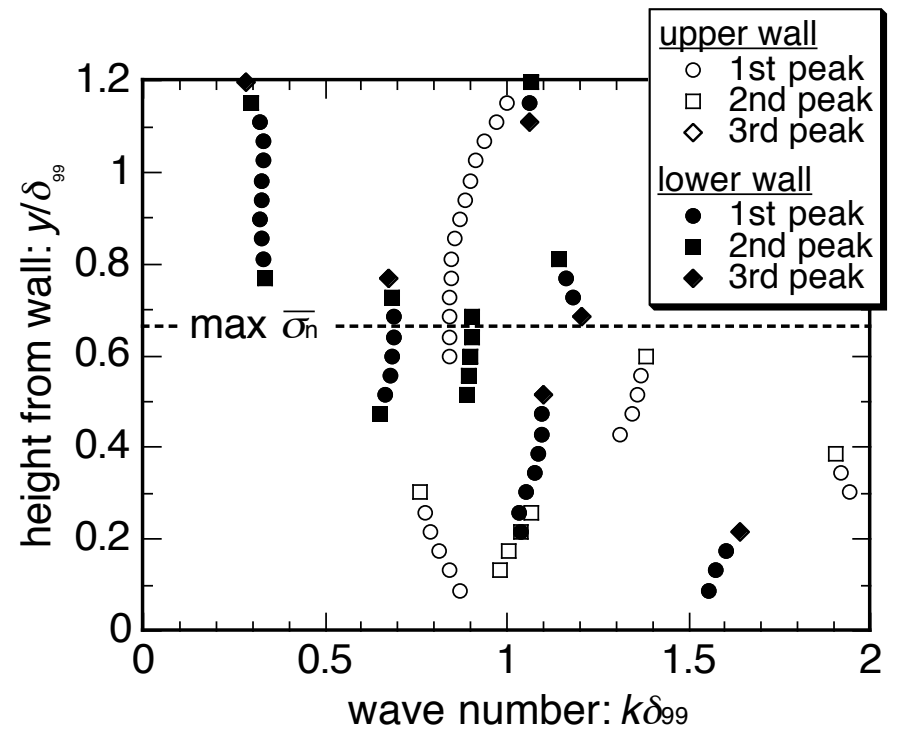

Fig. 14 Dominant scales of turbulent structures in a Mach 2 supersonic boundary layer.

Figure 14 shows the dominant scales of the turbulent structures in the boundary layer. The open symbols represent the upper wall, and the solid symbols represent the lower wall. The scale $k_{\mathrm{d}}$ was normalized by $1 / \delta_{99}$. In Figure 14 , the dominant scale in the boundary layer appears similar to $\delta_{99}$. However, the boundary layer contained not only structures having a similar scale to $\delta_{99}$ but also structures having other scales. The dominant scales near the outer edge of the boundary layer were larger than those near the wall. In the present case, the largest scale was approximately $3 \times$ $\delta_{99}$, which is similar to that at $x_{\text {nozzle }}=170-200 \mathrm{~mm}$ in Fig. 14 . On the other hand, the smallest scales were half of $\delta_{99}$. 


\subsection{Sonic Injection into Supersonic Crossflow}

For the transverse-jet case, the evaluation of $U_{\mathrm{C}}$ appeared to be significantly affected by the trajectory of the convection compared with that for the boundary-layer case, where the trajectory was almost parallel to the wall. Therefore, we checked the validity of the maximum $\overline{\sigma_{n}}$ trajectory as the trajectory of the convection. Figure 15 compares the maximum $\overline{\sigma_{n}}$ trajectory to the convection-velocity direction evaluated via a spatial cross-correlation-based method (Ben-Yakar \& Hanson 2001; Ben-Yakar et al. 2006). In the correlation-based method, an interrogation window whose size was selected to fully encompass a typical structure was employed, and the displacement of the structure between two time-separated frames was evaluated using a spatial cross-correlation algorithm such as PIV. Thus, we call this method an SIV.

For the correlation-based method, the structure displacements were evaluated by using a direct cross-correlation algorithm with sub-pixel estimation. The interrogation-window size was manually determined by viewing the schlieren movies and differed with respect to $x / D$, typically in the range of 11-31 pixels. The dashed enclosed regions in Fig. 15 show the interrogation window for the correlation-based method. The searching area within the interrogation area was \pm 3 pixels. To improve the accuracy of evaluating the displacement beyond the pixel resolution, the images were interpolated via Fourier interpolation (Suzuki et al. 2012) before taking the cross-correlation, which is similar to the zero-padding FFT mentioned previously. For the Fourier interpolation, the image was first converted by the 2D FFT, and then zeroes were appended in the frequency domain. After the zero-padding, the image in the frequency domain was inverted in the spatial domain.

The thick vectors in Fig. 15 are the mean vectors of the convection velocity evaluated using the correlation-based method. The dashed lines indicate the trajectory of interest evaluated using the $\overline{\sigma_{n}}$ image. Figure 15 shows that the maximum $\overline{\sigma_{n}}$ trajectory was tangential to the convectionvelocity direction. Therefore, the maximum $\overline{\sigma_{n}}$ trajectory is appropriate as the jet trajectory.

Figures 16 shows the convection velocity of the dominant structures in the jet and the boundary layer. The horizontal axis indicates the distance from the injector normalized with respect to $D$, and the vertical axis indicates the convection velocity normalized with respect to $U_{\infty}$. The symbols represent the data obtained using the proposed method, and the lines represent the data obtained using the correlation-based method. The error bars indicate the variation of data among the highest, second-highest, and third-highest peaks. For both the jet and the boundary 
layer, the convection velocities obtained using the proposed method agree well with those obtained using the correlation-based method.

For the jet, Figure 16 shows that the convective velocity rapidly increased for $x / D \leq 5$, where the jet was bended towards the streamwise direction by the freestream and reached $U_{\mathrm{C}} / U_{\infty}=1.2$ 1.3. It gradually decreased to $U_{\mathrm{C}} / U_{\infty} \approx 1$ at $x / D \approx 25$. The schlieren image shown in Fig. 3 reveals that the reattachment shock wave was located in the range of $x / D=10-20$. The gradual decrease in $U_{\mathrm{C}} / U_{\infty}$ for $\mathrm{x} / \mathrm{D}=5-25$ was caused by the reattachment shock wave. At $x / D \approx 40, U_{\mathrm{C}} / U_{\infty}$ decreased below $U_{\mathrm{C}} / U_{\infty}=1$ because the reflected bow shock wave impinged into the jet.

It was previously reported that large-scale structures rolled up at a convection speed close to the jet exit velocity $V_{\mathrm{j}}$ (Gruber et al. 1997; Ben-Yakar and Hanson 2002). The jet turned to the streamwise direction after traveling a certain $x / D$, and the convection velocity was relaxed towards the freestream velocity. Our measurements well captured the relaxation of $U_{\mathrm{C}}$ towards the freestream value. However, they did not reproduce $U_{\mathrm{C}} \approx V_{\mathrm{j}}$ near the injector. For the present study, the injectant was room-temperature helium; thus, the jet exit velocity $V_{\mathrm{j}}$ was $874 \mathrm{~m} / \mathrm{s}\left(V_{\mathrm{j}} / U_{\infty}=\right.$ 1.73). For both the zero-padding $2 \mathrm{D}$ FFT and the SIV, the convection velocity did not reach this value. We attribute this to the low resolution of the imaging system. Because our imaging system captured the entire flow region of the transverse injection over $x / D>60$, it was difficult for the system to capture the generation of the very small eddies in the vicinity of the injector.

Figure 17 shows the wave number of the dominant structures in the jet, which were normalized with respect to $1 / D$. The scale was approximately three-fold larger than $D$ for $x / D \leq 10$. This corresponds to the scale with $3 \mathrm{~mm}$. For $x / D>10$, the wave number rapidly decreased to $k D \approx$ 0.15 at $x / D \approx 30$. This means that the small structures $3 \mathrm{~mm}$ in size near the injector grew to a size of $6.5 \mathrm{~mm}$ at $x / D \approx 30$. For $x / D>30$, the development of the structures was stopped. After the reattachment shock passed the range of $x / D=10-20$, the convection speed of the turbulent structures decreased to the freestream value, and the velocity difference between the freestream and the jet-which was a driving force for the development of the structures-disappeared. Consequently, the turbulent structures stably convected downstream. For the transverse injection into a supersonic flow, the reattachment shock wave and the flow deflection appeared to dominate the scale of the structures in the jet. 


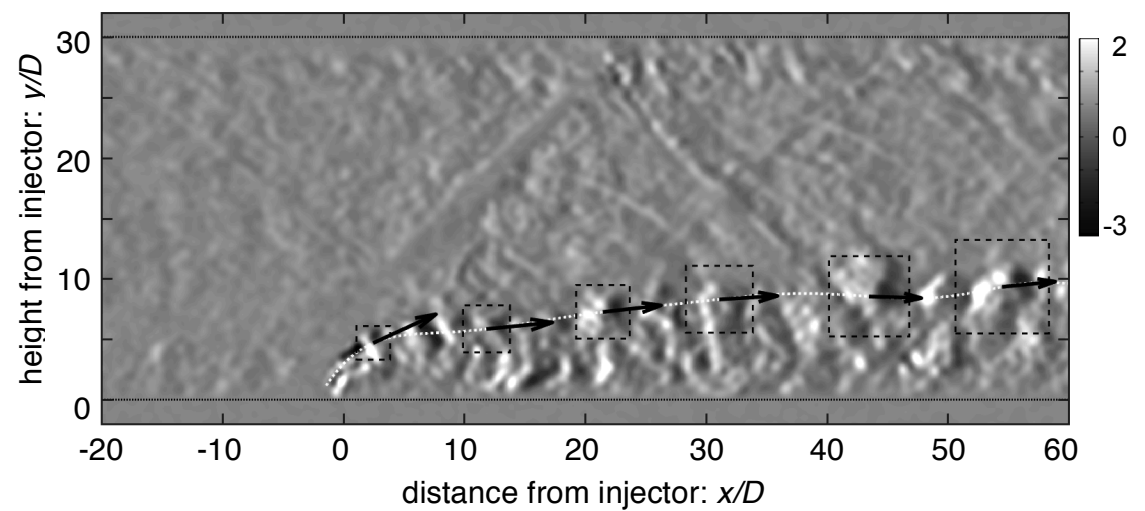

Fig. 15 Comparison of the maximum root-mean-square trajectory with the flow direction estimated using the correlation-based method.

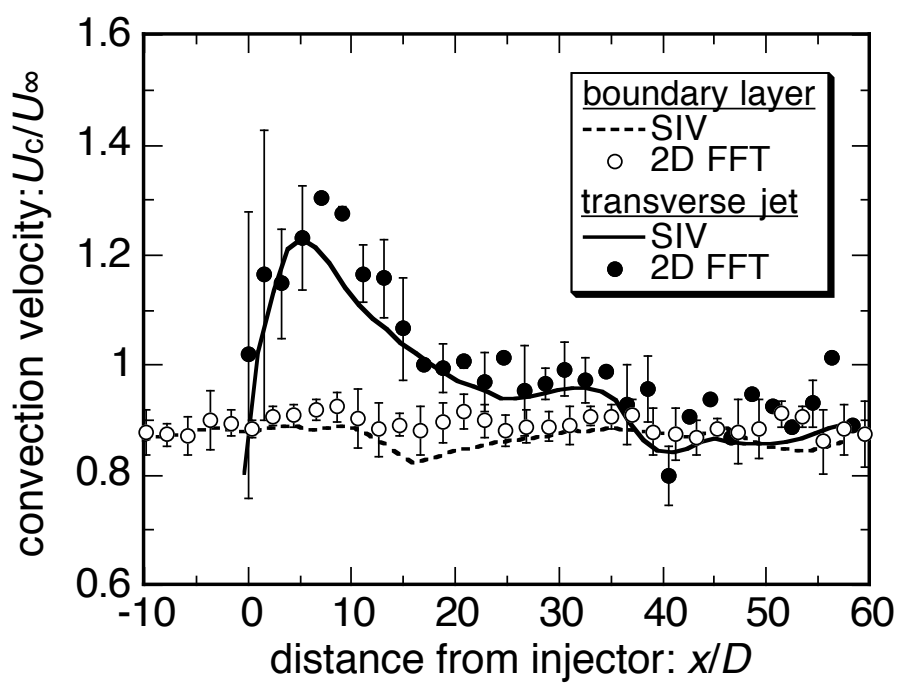

Fig. 16 Convection speeds of the turbulent structures within a transverse jet in a supersonic flow.

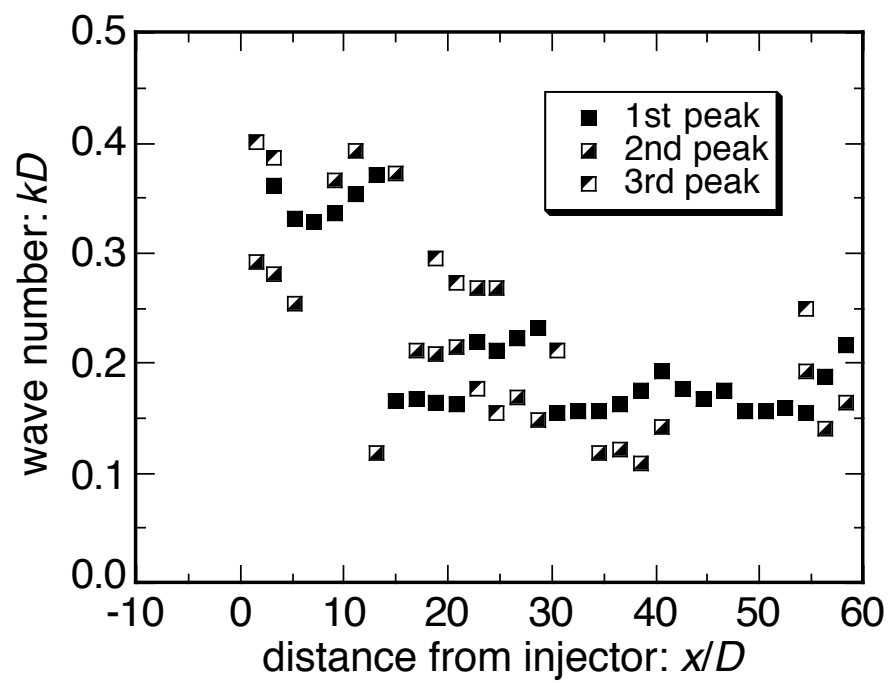

Fig. 17 Wave numbers of the dominant turbulent structures within a transverse jet in a supersonic flow. 


\section{Conclusion}

We captured time-series schlieren images by using a fast-framing camera with a frame rate of $1 \mathrm{MHz}$. The schlieren images revealed the turbulent structures within the boundary layer and the transverse jet into a Mach 2 supersonic flow. We developed a new image-analysis method using a 2D Fourier transform for efficiently evaluating the convection velocity and scale of the dominant structures.

In the schlieren images, the turbulent structures appeared as periodical gradations of the image intensity. First, the image intensities on the turbulent structures were sampled along the trajectory where the turbulent structures were convected, and a time-space intensity map was reconstructed from them. The time-space map showed right-running stripe patterns. The stripe patterns indicate that stable structures were periodically generated and convected downstream. When the Fourier transform was applied to the time-space map containing diagonal stripes, the transformed image exhibited high-amplitude points corresponding to both the angle and interval of the stripes in the time-space map. The convection velocity and dominant scale of the turbulent structures were calculated according to the angle and distance from the DC component to the high-amplitude point in the Fourier domain. The frequency-wave number resolution in the Fourier domain was improved by using a zero-padding FFT method. The accuracy of the peak estimation in the Fourier domain was improved using a sub-pixel peak-finding method with a Gaussian fit. The combination of the zero-padding method and the sub-pixel estimation allowed the accurate detection of highamplitude points even in cases of a short data length for the Fourier transform.

We applied this image-analysis method for both the supersonic boundary layer and the transverse jet into a Mach 2 supersonic flow. To validate the proposed method, the results were compared with those obtained using SIV. For both cases, the convection velocity obtained using the proposed method agreed well with those obtained using SIV.

For the supersonic boundary layer, the convective velocity was approximately $85 \%$ of the freestream velocity for entire region at $y / \delta_{99}>0.5$. This agrees with previously published experimental data. Our method revealed that various scales coexisted in the boundary layer. Near the outer edge of the boundary layer, the dominant scale was three times larger than the boundarylayer thickness. On the other hand, it was significantly smaller than the boundary-layer thickness near the wall. In addition to these structures, turbulent structures having a scale similar to the 
boundary-layer thickness coexisted across the boundary layer. The coexistence of the small- and large-scale structures indicates that some small structures produced large-scale motion in the boundary layer.

For the transverse jet, the analysis revealed that both the convective velocity and the dominant scale of the turbulent structures in the jet changed as the structures travelled downstream. Near the injector, the dominant scale was approximately three times larger than the injector diameter. The convection velocity rapidly increased to $x / D \approx 5$. These small structures merged with each other and rapidly formed much larger structures at $x / D \approx 15$. The scale was seven times larger than the injector diameter at $x / D \approx 30$. In the range of $x / D=15-30$, the convection velocity rapidly decreased and reached the freestream velocity. The schlieren images show that the reattachment shock wave was located here. Therefore, the reattachment shock wave and the flow deflection due to this shock wave appeared to dominate the development of the turbulent structures in the jet.

The two aforementioned examples indicate that the proposed image-analysis method is a powerful tool for understanding the motion of turbulent structures captured in fast-framing schlieren imaging. This analysis provides us quantitative information regarding the relationship between the convective velocity and dominant scale of the structures throughout their development process. The method has the potential to easily extract quantitative information from other fastframe images, such as PLIF and PLS, using a pulse-burst laser because the flow features in the images have periodical patterns.

Acknowledgments This work was supported by the Ministry of Education, Culture, Sports, Science and Technology in Japan, Grant-in-Aid for Young Scientist (B), 20760105 and JSPS KAKENHI Grant-in-Aid for Scientific Research (B) $15 \mathrm{H} 04199$.

\section{References}

Ben-Yakar A, Hanson RK (2002) Ultra-Fast-Framing Schlieren System for Studies of the Time Evolution of Jets in Supersonic Crossflows. Exp Fluids 32:652-666. DOI:10.1007/s00348002-0405-z

Ben-Yakar A, Mungal MG, Hanson RK (2006) Time Evolution and Mixing Characteristics of Hydrogen and Ethylene Transverse Jets in Supersonic Crossflow. Phys Fluid 18(2):026101-1026101-16. DOI:10.1063/1.2139684

Brown GL, Roshko A (1974) On Density Effects and Large Structure in Turbulent Mixing Layers. J Fluids Mech 64(4):775-816. DOI:10.1017/S002211207400190X 
Bueno PC, Ganapathisubramani B, Clemens NT, Dolling DS (2005) Cinematographic Planar Imaging of A Mach 2 Shock Wave Turbulent Boundary Layer Interaction. AIAA-2005-0441. DOI:10.2514/6.2005-441

Comte-Bellot G, and Sarma GR (2001) Constant Voltage Anemometer Practice in Supersonic Flows. AIAA J 39(2):261-270. DOI:10.2514/2.1321

Ganapathisubramani B, Clemens NT, Dolling DS (2006) Large-scale motions in a supersonic turbulent boundary layer. J Fluid Mech 556:271-282. DOI:10.1017/S0022112006009244

Ganapathisubramani B, Clemens NT, Dolling DS (2007) Effects of Upstream Boundary Layer on The Unsteadiness of Shock-induced Separation. J Fluid Mech 585:369-394. DOI: $10.1017 / \mathrm{S} 0022112007006799$

Gruber MR, Nejad AS, Chen TH, Dutton JC (1997) Large Structure Convection Velocity Measurements in Compressible Transverse Injection Flowfields. Exp Fluids 22(5):397-407. DOI: $10.1007 / \mathrm{s} 003480050066$

Hargather MJ, Lawson MJ, Settles GS (2011) Seedless Velocimetry Measurements by Schlieren Image Velocimetry. AIAA J 49(3):611-620. DOI:

Jonassen D, Settles GS, Tronosky MD (2006) Schlieren PIV for Turbulent Flows. Optics and Lasers in Engineering 44(3-4):190-207. DOI:10.1016/j.optlaseng.2005.04.004

Kim KC, Adrian RJ (1999) Very Large-Scale Motion in The Outer Layer. Phys Fluids 11(2): 417-423. DOI:10.1063/1.869889

Kondo A, Hayase H, Sakaue S, Arai T (2009) Effect of Expansion Ramp Angle on Supersonic Mixing using Streamwise Vortices. AIAA-2009-7314. DOI:10.2514/6.2009-7314

Kondo A, Sakaue S, Arai T (2010) Measurement Method of Mass Flux and Concentration in Supersonic Mixing by Hot-Wire Anemometry. International Journal of Emerging Multidisciplinary Fluid Sciences 2(1):15-26. DOI:10.1260/1756-8315.2.1.15

Lourenco L, Krothapalli A (1995) On the Accuracy of Velocity and Vorticity Measurements with PIV. Exp Fluids 18:421-428.

Poggie J, Erbland PJ, Smits AJ Miles RB (2004) Quantitative Visualization of Compressible Turbulent Shear Flows Using Condensate-enhanced Rayleigh Scattering. Exp Fluids 37:438-454. DOI:10.1007/s00348-004-0828-9.

Poggie J, Leger T (2015) Large-scale Unsteadiness in A Compressible, Turbulent Reattaching Shear Layer. Exp Fluids 56:205. DOI:10.1007/s00348-015-2064-x.

Smith MW, Smits AJ (1995) Visualization of the Structure of Supersonic Turbulent Boundary Layer. Exp Fluids 18:288-302. DOI:10.1007/bf00195099

Uramoto S, Kouchi T, Masuya G (2016) Effects of Upstream Disturbances on Supersonic Flowfield with Transverse Injection. Journal of the Japan Society for Aeronautical and Space Sciences 64(4):244-252. DOI:10.2322/jjsass.64.244

Thurow B, Jiang N, Lempert W (2013) Review of ultra-high repetition rate laser diagnostics for fluid dynamic measurements. Measurment Science and Technology 24:012002. DOI:10.1088/0957-0233/24/1/012002

Willert C, Gharib M (1991) Digital Particle Image Velocimetry. Exp Fluids 10(4):182-193. DOI:10.1007/BF00190388 